\title{
Parent-Reported Outcomes of Early Childhood Selective Dorsal Rhizotomy for the Treatment of Spastic Diplegia
}

\author{
TS Park ${ }^{1}$, Susan Joh ${ }^{1}$, Deanna M. Walter ${ }^{1}$, Nicole L. Meyer ${ }^{1}$ \\ 1. Pediatric Neurosurgery, Washington University School of Medicine, St. Louis Children's Hospital, St. Louis, USA
}

Corresponding author: TS Park, tspark@wustl.edu

\section{Abstract \\ Background}

A selective dorsal rhizotomy (SDR) is employed to treat spastic cerebral palsy. The surgical techniques and patient care protocols vary among hospitals. One of the variations is the age cut-off for SDR. We have been advocating SDR to be performed early - especially at ages 2 and 3. With this study, we are reporting the feasibility and parent-reported surgical outcomes of receiving SDR at an early age for the treatment of spastic diplegia.

\section{Objectives}

Our aim is to examine the safety and benefits of receiving SDR at the ages of 2 and 3 for the treatment of spastic diplegia.

\section{Methods}

The Institutional Review Board (IRB) of Washington University School of Medicine approved this retrospective quality of life survey and chart review (approval \#202009056). The subjects of this study were children and teens (ages: 3.9-18.1) with spastic diplegic cerebral palsy who underwent SDR at ages 2 or 3 between years 2005 and 2019 at St. Louis Children's Hospital. Only domestic patients that were minors at the time of the study were selected to be participants in compliance with IRB regulations to protect patient health information that could potentially be breached by sending information to an incorrect or dated email. Thus, all contact was made through postal mail. The study included 141 patients from a total of 362 eligible patients. Parents of eligible patients were sent the research survey via postal mail. Only patients who responded to the survey were included in this study. The survey included questions on demographic information, quality of life, health perception, motor and ambulatory functions, braces and orthotics, pain issues, side effects of SDR, and post-SDR treatment.

Review began 05/09/2021 Review ended 06/01/2021 Published 06/08/2021

\section{(๑) Copyright 2021}

Park et al. This is an open access article distributed under the terms of the Creative Commons Attribution License CC-BY 4.0., which permits unrestricted use, distribution, and reproduction in any medium, provided the original author and source are credited.

\section{Results}

The study included 141 diplegic patients. Of all patients at the time of the study, 91\% reported an improvement in walking, $92 \%$ in standing, and $89 \%$ in sitting. In daily life activities, $87 \%$ of patients became more independent after SDR. $65 \%$ of patients were able to walk without a walking aid and about $4 \%$ were not able to walk. $11 \%$ of all patients relied mostly on a wheelchair. Moreover, $43 \%$ of patients were able to run independently. Regarding post-SDR orthopedic surgery, $48 \%$ of patients received at least one type of orthopedic surgery, with Achilles tendon lengthening, hamstring lengthening, and calf muscle release being the most common types.

\section{Conclusions}

SDR performed at an early age through a single-level laminectomy was proved feasible and safe. A follow-up until the adult age (18 years) showed improvements in walking and other motor functions. The results support the implementation of early-age SDR for the treatment of spastic diplegia.

Categories: Neurology, Pediatric Surgery, Neurosurgery

Keywords: children, early selective dorsal rhizotomy, spastic diplegia, spasticity, quality of life, pediatric orthopedic surgery

\section{Introduction}

Selective dorsal rhizotomy (SDR) is employed around the world to treat spastic cerebral palsy (CP). The surgical techniques of SDR and overall patient care methods have evolved over the last three decades [1-5]. Individual centers have adopted different protocols in their practices, with age cut-offs for SDR being one of the differences. In St. Louis Children's Hospital, we have been advocating receiving SDR at an early age (2 and 3 years) for many years. We report the feasibility of early-age SDR and parent-reported surgical 


\section{Materials And Methods}

The Institutional Review Board (IRB) of Washington University School of Medicine approved this retrospective quality of life survey and chart review (approval \#202009056). The subjects of this study were children and teens (ages: 3.9-18.1) with spastic diplegic CP who underwent SDR at ages 2 or 3 (up to the fourth birthday) between years 2005 and 2019 at St. Louis Children's Hospital. Only domestic patients who were minors at the time of the study were selected to be participants in compliance with IRB regulations to protect patient health information that could potentially be breached by sending information to an incorrect or dated email. Therefore without prior in-person consent to email patients for research recruitment, all contact was made through postal mail. International patients were not recruited as using postal mail with these patients was not possible; we did not have their full address. We gathered contact information from our clinic's database and medical records, which only includes the mailing addresses of the patients' parents or guardians. Only minors were recruited because parents and guardians are fully able to consent for a minor and access a minor's health information. Consent was obtained from the patients' guardians by filling out the survey and sending it back.

The survey was constructed with questions on demographic information, perception of health, quality of life, pre and postoperative motor and ambulatory functions, post-SDR treatment, braces and orthotics, side effects of SDR, and perception of the SDR procedure. Demographic information included the date of birth, living situation, and current student and employment status.

A five-point scale from poor to excellent was utilized to evaluate the perception of the patients' health, as seen in Question 1 of the SF-36 health survey. Yes/no questions were used to indicate improvement due to SDR in abilities in walking, standing, sitting, ability to exercise, ability to stretch, and endurance.

Yes/no questions were used to evaluate the quality of life. Questions included receiving help with eating, hygiene, bladder, and transferring positions. Questions were asked about the patients' independence after SDR, engagement in recreational sports, and regularity of strengthening and stretching muscles.

The Gross Motor Function Classification Scale (GMFCS) was used to construct questions assessing patients' pre- and post-SDR motor function including running, walking, and sitting. Different questions were used for different age groups (2-3, 4-5, 6-11, and 12-18 years) for the differences in each stage of motor development. Separate questions were included to assess pre- and post-SDR ambulatory function by asking patients to indicate their level of function based on a five-level classification system. Level 1 includes patients who can run independently and level 5 includes patients who cannot walk.

Questions about bladder function, pain, and loss of sensation in the lower limbs were asked to assess the side effects of SDR. Participants who indicated numbness or loss of sensation were asked to further indicate the location of the numbness. Patients were asked to provide any other negative side effects if present. PostSDR questions about scoliosis, post-SDR orthopedic surgical interventions, medications for spasticity, and Botox were also inquired.

Yes/no/unsure questions were used to ask patients if they had benefited from SDR and if they would recommend the procedure to other patients. They were also given the option to add any additional comments about their perception of SDR.

\section{Results}

\section{Study cohort}

The total number of patients diagnosed with diplegic CP who received an SDR at ages 2 and 3 (up to the fourth birthday) during the study period (2005-2019) is 744 patients. Participants chosen for this research study included only domestic patients who were minors and followed after SDR for at least one year; international patients and those above the age of 18 years were excluded in compliance to IRB regulations to protect patient health information that could be breached by sending information to an incorrect email. The IRB required that all patients be contacted only through postal mail and not email as we did not have prior in-person consent to email them specifically for research recruitment. The research team identified patients who qualified for the study - domestic patients with updated residential addresses - and gathered contact information from the Center for Cerebral Palsy Spasticity at St. Louis Children's Hospital's database. Of the 744 patients, we found a total of 362 eligible patients and received 141 responses, resulting in a 39\% response rate. All patients underwent a single-level laminectomy SDR as described in an earlier publication $[6]$.

\section{Demographics of the study cohort}

The participants' ages were 2.1 to 4.0 years (mean age: $3.1 \pm 0.5$ ) at the time of surgery (Table 1 ). When they completed the survey, the patients were 3.9 to 18.1 years (mean age: $9.7 \pm 4.0$ ). All ages are considered the 


\section{Cureus}

patient's current age (i.e. age 5.9 is 5 years old). The follow-up period, referring to the period between the date of surgery and the date of the follow-up study, ranged from 1.0 to 15.2 years. In that, $57 \%$ of participants identified as male and $43 \%$ identified as female and $97 \%$ of patients were attending school at the time of the survey.

The $3 \%$ of patients who were not attending school were 5 years or younger. However, 25 patients that were 5 years or younger at the time of completing the survey were attending school. Eleven patients (ages: 3-10) were attending school part-time. This survey took place during the COVID-19 pandemic and may have affected this data. Four percent of patients were working, all part-time (ages: 14-17).

Study Population

Total No. of Patients

Age at surgery

Age at follow-up survey

Follow-up period

Sex

Male

Female

Education

Currently attending school

\section{1 patients}

2.1-4.0 years $(3.1 \pm 0.5$, mean $\pm S D)$

3.9-18.1 years $(9.7 \pm 4.0$, mean $\pm S D)$

$1.0-15.2$ years $(6.5 \pm 4.0$, mean $\pm S D)$

$\%$ of patients

57

43

$\%$ of patients

97

TABLE 1: Demographics of 141 diplegic patients at the time of the survey

\section{Health perception after SDR}

Almost all patients (96\%) reported a health perception of good or better. In that, $44 \%$ of patients reported "Excellent", 45\% reported "Very good", and 7\% reported "Good". Only 1\% reported "Fair" and none reported "Poor". Three patients had no answer for health perception, and two of those patients had no answers for the entire section (including improvements) (Table 2).

A majority of the patients reported improvements in each of the categories questioned: walking (91\%), standing (92\%), sitting (89\%), balance when walking (79\%), ability to exercise (86\%), ability to stretch (92\%), endurance (79\%), and recreational sports (60\%). Most frequent improvements were seen in the ability to stretch, stand, and walk. Not every patient had answers to every question. 


\section{Cureus}

\begin{tabular}{|c|c|c|c|}
\hline Health Perception & \multicolumn{3}{|c|}{$\%$ of patients } \\
\hline Excellent & \multicolumn{3}{|l|}{44} \\
\hline Very good & \multicolumn{3}{|l|}{45} \\
\hline Good & \multicolumn{3}{|l|}{7} \\
\hline Fair & \multicolumn{3}{|l|}{1} \\
\hline Poor & \multicolumn{3}{|l|}{0} \\
\hline No response & \multicolumn{3}{|l|}{2} \\
\hline \multicolumn{4}{|c|}{ Improved Motor Functions ${ }^{\star}$} \\
\hline & Yes (\%) & No (\%) & Unsure (\%) \\
\hline Walking & 91 & 6 & 1 \\
\hline Standing & 92 & 6 & 0 \\
\hline Sitting & 89 & 9 & 1 \\
\hline Balance when walking & 79 & 16 & 1 \\
\hline Ability to exercise & 86 & 11 & 1 \\
\hline Ability to stretch & 92 & 6 & 0 \\
\hline Endurance & 79 & 17 & 1 \\
\hline Recreational sports & 60 & 33 & 0 \\
\hline
\end{tabular}

TABLE 2: Health and physical ability improvement perception after SDR of 141 diplegic patients

*Not all patients responded to all questions.

\section{Independence in daily physical activities}

Patients were separated into age groups as children typically require help in several physical activities when they are younger. Thirty patients (21\%) were aged 4 to 5,61 patients $(43 \%)$ were aged 6 to 10 , and 50 patients (36\%) were aged 11 to 18 (Table 3).

Patients aged 4 and 5 were not included in this section because children in this age group typically require help in several aspects of daily life. Children typically begin to become more independent at age 6 .

For patients aged 6 to 10, one patient had no answers to all questions in this section. One patient (1\% overall) indicated that they sometimes have help getting dressed and using the toilet. This patient was included with others that answered "yes" to requiring help with those activities.

For patients aged 11 to 18 , one patient had no answer for requiring help during eating, and another had no answer for requiring help getting dressed.

From the data, a general trend of more independence in daily activities can be seen, yet this trend is likely due to normal aging as all patients are at least a year post-treatment. To account for this possibility, questions were asked about improvement in independence after SDR. 87\% of all patients reported improvement in independence overall, $11 \%$ reported no change, and $2 \%$ did not answer. One patient who had no answer commented that improvement in independence was hard to judge as the improvements may have been from the natural course of growing up. Two patients believed their course of ability to walk worsened after SDR. 


\section{Cureus}

\begin{tabular}{|c|c|c|c|c|}
\hline \multicolumn{5}{|l|}{ Daily Activities } \\
\hline \multirow[t]{2}{*}{ Requires: } & \multicolumn{4}{|c|}{$\%$ of patients* ** } \\
\hline & \multicolumn{2}{|c|}{ Ages 6-10 } & \multicolumn{2}{|c|}{ Ages 11-18 } \\
\hline \multirow[t]{2}{*}{ (\# of patients) } & \multicolumn{2}{|c|}{61 patients } & \multicolumn{2}{|c|}{50 patients } \\
\hline & Yes & No & Yes & No \\
\hline Help eating & 11 & 87 & 10 & 88 \\
\hline Help using the toilet & 38 & 61 & 28 & 72 \\
\hline Help getting dressed & 59 & 39 & 32 & 66 \\
\hline Exercises regularly & 93 & 3 & 78 & 22 \\
\hline Stretches legs regularly & 80 & 18 & 66 & 34 \\
\hline Plays recreational sports & 33 & 66 & 34 & 66 \\
\hline
\end{tabular}

TABLE 3: Independence in daily physical activities by age group of 141 diplegic patients

${ }^{*}$ Percentage is out of the number of patients in the categorized age group.

**30 patients aged 4-5 were left out as most patients required help in all activities because of age.

\section{Gross motor functions before and after SDR}

Different age groups were asked different questions about gross motor functions according to their developmental stage. Questions constructed for this section were based on the Gross Motor Function Classification System (GMFCS) [7]. Pre-SDR gross motor function questions were based on abilities typical for 2 and 3-year-olds. Before SDR, 55\% of patients preferred creeping and crawling, 57\% cruised while holding onto furniture, and $46 \%$ required help steering when walking. For sitting ability, $45 \%$ of patients stood and sat without help from an adult (Table 4).

Post-SDR questions were more similar across age groups as children should be able to walk by age 4 ; therefore, patients were not separated into age groups for this section. In that, $65 \%$ of patients were able to walk without a walking aid and $32 \%$ were able to walk with a walking aid, totaling $97 \%$ that can walk (Table 4). However, $4 \%$ of patients indicated that they do not walk (Table 5). Of the patients that do walk, $39 \%$ indicated that they can walk on all surfaces and $57 \%$ have difficulty walking on uneven surfaces. Forty-six percent of patients use a wheelchair for long distances or outside including $11 \%$ of all patients that mostly rely on a wheelchair. Also, $92 \%$ can sit and stand without help (previously $45 \%$ ) (Table 4).

GMFCS is a classification system in which the gross motor function of children and teens with CP are categorized into five different levels. Level I is the highest functioning category and level V is the lowest. Pre-SDR GMFCS levels were found from our physical therapy records. Post-op levels were also found from physical therapy records but were mostly determined by the survey responses as the survey was the most recent measure of gross motor function. All $26 \%$ of patients with a pre-op GMFCS level I also had a post-SDR GMFCS of level I; $45 \%$ of patients classified as level I postoperatively. All patients were at least level IV both before and after SDR; $12 \%$ were level IV before the surgery and only 9\% were level IV after (Table 4). 


\section{Cureus}

\begin{tabular}{|c|c|c|}
\hline \multirow[t]{2}{*}{ Gross Motor Functions } & \multicolumn{2}{|c|}{$\%$ of patients } \\
\hline & Pre-SDR & Post-SDR \\
\hline \multicolumn{3}{|l|}{ Walkıng } \\
\hline Prefers creeping and crawling & 55 & - \\
\hline Cruises holding furniture & 57 & - \\
\hline Help steering when walking & 46 & - \\
\hline Walks (no walking aid) & - & 65 \\
\hline Walks only with walking aid & - & 32 \\
\hline Can use stairs (no handrails) & - & 23 \\
\hline Able to walk on all surfaces & - & $39^{*}$ \\
\hline Difficulty walking on uneven surfaces & - & $57^{*}$ \\
\hline Wheelchair for long distances or outside & - & 46 \\
\hline \multicolumn{3}{|l|}{ Sitting } \\
\hline Stands and sits (no help) & 45 & 92 \\
\hline Wheelchair (home, school, community) & - & 11 \\
\hline \multicolumn{3}{|l|}{ GivirCS Level } \\
\hline 1 & 26 & 45 \\
\hline II & 33 & 23 \\
\hline III & 30 & 23 \\
\hline IV & 12 & 9 \\
\hline
\end{tabular}

TABLE 4: Gross motor functions in different age groups of 141 diplegia patients before and after SDR

Gross motor functions with a dash (-) were those not applicable to the age group and therefore were not asked.

${ }^{\star}$ Four percent of patients do not walk.

Ambulatory Function

Walks without mobility device in all environments

Walks without mobility device in protected environments

Walks with crutches or canes

Walks with walker

Does not walk

Running

Runs independently

\section{$\%$ of patients}

Pre-SDR

Post-SDR

14

52

$13 \quad 13$

3

15

$52 \quad 16$

18

4

3

43

TABLE 5: Ambulatory function of 141 diplegic patients before and after SDR 


\section{Ambulatory function after SDR}

SDR procedure proved to be beneficial to many patients (Table 5). All patients who could run independently before the procedure were able to run independently after. Fourteen patients walked without a mobility device in all environments, 14 patients walked without a mobility device in protected environments, 21 patients walked with a walker, three patients walked with crutches or canes, and four patients did not walk could run independently after. It is important to note that some of the children in this study were under 2 years old and all were at most age 4.0 before SDR. At this age, children are still developing their ability to walk. For this reason, questions about walking ability were not asked in the previous section. However, because many children start walking at age 2 , questions were asked about ambulatory function before and after SDR to compare the change in ability.

All but one patient that was not able to walk before SDR could not walk after. The patient that could not walk after the procedure indicated that they walked with a walker before SDR.

\section{Orthopedic surgery after SDR}

Orthopedic surgery is a crucial procedure for patients with spastic $\mathrm{CP}$ with muscle contractures and is performed on patients to release muscle tightness. SDR allows for these patients to have a minimally invasive muscle-tendon lengthening orthopedic procedure as it reduces spasticity. For this patient cohort, $48 \%$ of patients reported receiving orthopedic surgery; $29 \%$ had only one type of orthopedic surgery, and $18 \%$ had two or more types. Two patients indicated receiving orthopedic surgery but did not specify the type of surgery. Achilles tendon lengthening (21\%), hamstring lengthening (18\%), and calf muscle release (16\%) were the most common orthopedic surgeries (Table 6). It is important to note that the need for orthopedic surgery is not a result of SDR. The high incidence of orthopedic surgery is due in part to our practice preference for performing early orthopedic surgery in children 5 years old and older. Another reason is the fact that due to motor impairment, many diplegic children have limited knee extension and foot dorsiflexion while walking. As a result, the hamstrings and calf muscles are not able to fully stretch during daily activities, and muscle contractures develop even without spasticity. The restricted knee extension and foot dorsiflexion may persist even after SDR causing limited hamstring and calf muscle flexibility when walking and participating in other physical activities, thus requiring orthopedic intervention. With this in mind, monitoring children closely after SDR to detect early deformities is essential.

A small percentage of patients (6\%) were diagnosed with scoliosis. One indicated that the scoliosis was very minor and another that they were on the "watch list" but their physician was pleased. Only one patient received a spine fusion for their scoliosis (Table 6).

\begin{tabular}{|l|l|}
\hline Treatment & $\%$ of patients \\
\hline Orthopedic surgery* & 7 \\
\hline Adductor release & 18 \\
\hline Hamstring lengthening & 16 \\
\hline Calf muscle release & 21 \\
\hline Achilles tendon lengthening & 9 \\
\hline Hip surgery & 1 \\
\hline Spine fusion for scoliosis & 1 \\
\hline Current Medications & 4 \\
\hline Baclofen & 1 \\
\hline Botox & \\
\hline
\end{tabular}

\section{TABLE 6: Treatment after SDR in 141 diplegic patients}

*Each patient may have had more than one type of surgery.

\section{Discussion}

This is a retrospective non-controlled study in which data were collected using a survey from a portion of the potential study cohort. The study was conducted under the new constraining rules of the IRB. We were barred from contacting patients' parents by email and were required to contact parents only by postal mail. 
This limitation required us to exclude international patients and many domestic patients as we did not have postal addresses for these patients. We were also restricted to mail only minors, as they are under the protection of their parents or guardian, meaning that patient-protected information is accessible to parents and guardians. Thus, we were required to exclude patients who reached adult age. Notwithstanding the unprecedented limitations, we analyzed the feasibility, safety, potential benefits, and adverse effects of early childhood SDR. We expect that the results of our study will expand the strength of clinical experience with SDR for the treatment of spastic diplegia in children.

Most importantly, we provide evidence that the single-level laminectomy SDR on 2 and 3-year-old children are feasible and safe. In the study participants, we did not encounter postoperative complications such as a spinal fluid leak, paralysis, urinary incontinence, or surgical site infection requiring intravenous antibiotics. In patients who are near the end of childhood, we found no cases of SDR-related spinal problems or significant sensory loss. Likewise, none of the patients experienced a return of spasticity. Based on the results of an earlier study conducted on adult patients who had SDR in childhood [2,3], we are expecting that the safety of SDR will hold up later in adult life.

In able-bodied children, a mature gait pattern is established by 3 years of age, and their adult gait pattern is established by 7 years [8]. Beyond age 7, motor coordination is further refined by practice. Children with spastic CP are born with normal muscles. In late infancy, spasticity becomes symptomatic and begins to exert negative effects on the muscles. Spasticity damages muscles; it also decreases voluntary movements and associated muscle flexibility. Spasticity inhibits longitudinal muscle growth [9-11]. Muscle contractures and bony deformities develop due to the effects of spasticity and reduced active movements [12]. It is thus possible that early intervention of reducing spasticity with SDR would be more beneficial than late intervention.

Furthermore, we found that after 4 years of age, non-surgical treatment cannot reverse the established muscle contractures, and orthopedic surgery becomes the only treatment option. However, removing the patient's spasticity through SDR enables a minimally invasive muscle-tendon lengthening orthopedic surgery to treat the contractures [13]. It is noteworthy that nearly half of the participants in our study received orthopedic surgery despite early-age SDR. The high incidence of orthopedic surgery is due in part to our practice preference for performing early orthopedic surgery in children ages 5 and older. Another reason is the fact that due to motor impairment, many diplegic children have limited knee extension and foot dorsiflexion while walking. As a result, the hamstrings and calf muscles are not able to fully stretch during daily activities, and muscle contractures develop even without spasticity. The restricted knee extension and foot dorsiflexion may persist even after SDR causing limited hamstring and calf muscle flexibility when walking and participating in other physical activities. With this in mind, monitoring children closely after SDR to detect early deformities is essential.

Gross motor function and ambulatory function improved after SDR (Tables 4 and 5). Due to the granular data of each age group and the lack of a control group, we cannot determine the significance of the role of earlyage SDR in improving postoperative motor function. Nevertheless, the results are promising in that the percentage of patients who can walk independently increased from $14 \%$ pre-surgery to $52 \%$ post-surgery, and those able to run increased from $3 \%$ to $43 \%$. The improvements observed in overall motor functions and the absence of adverse effects from surgery suggest that early-age SDR for treatment of spastic diplegia is a viable option.

\section{Conclusions}

One hundred forty-one children underwent SDR at 2 and 3 years of age for spastic diplegia. No post-surgical complications were reported in the participants following an early-age SDR performed through a single-level laminectomy, confirming the safety of the procedure. The follow-up surveys of the patients revealed improvements in walking and other motor functions. The results support the intervention of early-age SDR for the treatment of spastic diplegia.

\section{Additional Information \\ Disclosures}

Human subjects: Consent was obtained or waived by all participants in this study. Institutional Review Board of Washington University School of Medicine issued approval 202009056. Animal subjects: All authors have confirmed that this study did not involve animal subjects or tissue. Conflicts of interest: In compliance with the ICMJE uniform disclosure form, all authors declare the following: Payment/services info: All authors have declared that no financial support was received from any organization for the submitted work. Financial relationships: All authors have declared that they have no financial relationships at present or within the previous three years with any organizations that might have an interest in the submitted work. Other relationships: All authors have declared that there are no other relationships or activities that could appear to have influenced the submitted work.

\section{References}


1. Mittal S, Farmer JP, Al-Atassi B, et al.: Long-term functional outcome after selective posterior rhizotomy . J Neurosurg. 2002, 97:315-25. 10.3171/jns.2002.97.2.0315

2. Park T, Liu JL, Edwards C, Walter DM, Dobbs MB: Functional outcomes of childhood selective dorsal rhizotomy 20 to 28 years later. Cureus. 2017, 9:e1256. 10.7759/cureus.1256

3. Park T, Edwards C, Liu JL, Walter DM, Dobbs MB: Beneficial effects of childhood selective dorsal rhizotomy in adulthood. Cureus. 2017, 9:e1077. 10.7759/cureus.1077

4. Peacock WJ, Staudt LA: Functional outcomes following selective posterior rhizotomy in children with cerebral palsy. J Neurosurg. 1991, 74:380-5. 10.3171/jns.1991.74.3.0380

5. Steinbok P: Long-term effect of selective dorsal rhizotomy . Dev Med Child Neurol. 2013, 55:586. 10.111/dmcn.12146

6. Park TS, Johnston JM: Surgical techniques of selective dorsal rhizotomy for spastic cerebral palsy. Technical note. Neurosurg Focus. 2006, 21:7e.

7. GMFCS Family Report Questionnaire. (2007). Accessed: May 3, 2021: https://canchild.ca/system/tenon/assets/attachments/000/000/481/original/GMFCS_Family.pdf.

8. Gait and Motor Milestones. (2021). Accessed: May 3, 2021: http://www.pmmonline.org/page.aspx?id=753.

9. de la Tour EH, Tabary JC, Tabary C, Tardieu C: The respective roles of muscle length and muscle tension in sarcomere number adaptation of guinea-pig soleus muscle. J Physiol (Paris). 1979, 75:589-92.

10. Williams PE, Goldspink G: The effect of immobilization on the longitudinal growth of striated muscle fibres . J Anat. 1973, 116:45-5.

11. Ziv I, Blackburn N, Rang M, Koreska J: Muscle growth in normal and spastic mice . Dev Med Child Neurol. 1984, 26:94-9. 10.1111/j.1469-8749.1984.tb04412.x

12. Hoffer MM, Knoebel RT, Roberts R: Contractures in cerebral palsy. Clin Orthop Relat Res. 1987, 70-7.

13. Limpaphayom N, Stewart S, Wang L, Liu J, Park TS, Dobbs MB: Functional outcomes after selective dorsal rhizotomy followed by minimally invasive tendon lengthening procedures in children with spastic cerebral palsy. J Pediatr Orthop Part B. 2020, 29:1-8. 10.1097/BPB.0000000000000642 